\title{
Photo-Induced Topological Phase Transition and a Single Dirac-Cone State in Silicene
}

\author{
Motohiko Ezawa \\ Department of Applied Physics, University of Tokyo, Hongo 7-3-1, 113-8656, Japan
}

\begin{abstract}
Silicene (a monolayer of silicon atoms) is a two-dimensional topological insulator (TI), which undergoes a topological phase transition to a band insulator under external electric field $E_{z}$. We investigate a photo-induced topological phase transition from a TI to another TI by changing its topological class by irradiating circular polarized light at fixed $E_{z}$. The band structure is modified by photon dressing with a new dispersion, where the topological property is altered. By increasing the intensity of light at $E_{z}=0$, a photo-induced quantum Hall insulator is realized. Its edge modes are anisotropic chiral, in which the velocities of up and down spins are different. At $E_{z}>E_{\mathrm{cr}}$ with a certain critical field $E_{\mathrm{cr}}$, a photo-induced spin-polarized quantum Hall insulator emerges. This is a new state of matter, possessing one Chern number and one half spin-Chern numbers. We newly discovered a single Dirac-cone state along a phase boundary. A distinctive hallmark of the state is that one of the two Dirac valleys is closed and the other open.
\end{abstract}

Introduction: Topological insulator (TI) is a distinctive state of matter indexed by topological numbers, and characterized by an insulating gap in the bulk and topologically protected gapless edges ${ }^{1,2}$. The topological classification has been applied to static systems ${ }^{3}$, but recently extended to timeperiodic systems 4 $^{-9}$. A powerful method to drive quantum systems periodically is to apply electromagnetic radiation to them. It can rearrange the band structure and change material properties by photon dressing. TIs may be obtained from a semimetal ${ }^{\frac{5}{4}}$ and from a band insulator ${ }^{8}$ (BI) in this way. Topological band structures may well be engineered by application of coherent laser beam in graphene and semiconductors. In this paper we propose a new type of topological phase transition in silicene, that is a photo-induced transition from a TI to another TI by changing its topological class.

Silicene, being synthesized ${ }^{10-12}$ only recently, is gifted with enormously rich physics ${ }^{13}-18$. Silicene consists of a honeycomb lattice of silicon atoms with buckled sublattices made of A sites and B sites. The states near the Fermi energy are $\pi$ orbitals residing near the $\mathrm{K}$ and $\mathrm{K}$ ' points at opposite corners of the hexagonal Brillouin zone. The low-energy dynamics in the $\mathrm{K}$ and $\mathrm{K}$ ' valleys is described by the Dirac theory as in graphene. However, Dirac electrons are massive due to a relatively large spin-orbit (SO) coupling $\lambda_{\mathrm{so}}=3.9 \mathrm{meV}$ in silicene. It is remarkable that the mass can be controlled ${ }^{14,15}$ by applying the electric field $E_{z}$ perpendicular to the silicene sheet.

Silicene is a quantum spin Hall insulator ${ }^{13}$ (QSHI), which is a particular type of TI. It undergoes a topological phase transition ${ }^{14,15}$ to a BI as $\left|E_{z}\right|$ increases and crosses the critical field $E_{\text {cr. }}$. We investigate photo-induced topological phase transition. Under the off-resonance coherent laser beam, Berry curvatures in the momentum space, originating from the SO coupling, are modified in the photon-dressed bands so that the occupied electronic states change topological properties ${ }^{4}$. The phase diagram has a remarkably rich structure, where there are three distinct topological phases in addition to one trivial phase.

We show that, by applying strong circular polarized light with frequency $\Omega$ at fixed $E_{z}$, silicene is transformed from a QSHI or a BI into a photo-induced spin-polarized quan- tum Hall insulator (PS-QHI) and eventually into a photoinduced quantum Hall insulator (P-QHI). Here, PS-QHI is a new state of matter indexed by one Chern and one half spinChern numbers. On the other hand, the edge modes of P-QHI are anisotropic chiral, where the velocities of up and down spins are different. Furthermore, there appear spin polarized metal (SPM) and spin valley-polarized metal ${ }^{16}$ (SVPM) on the crossing points of the two phase boundaries. They have different spin configurations. A particularly intriguing state appears along a phase boundary, which has only one closed gap with a linear dispersion. We call it the single Dirac-cone (SDC) state. It is utterly a new state as far as we are aware of. The electric field breaks inversion symmetry, while the light breaks time-reversal symmetry. When they are both broken, the gap can be different at $\mathrm{K}$ and $\mathrm{K}$ ' points. We comment that the Nielsen-Ninomiya theorem ${ }^{19}$, which states that all massless Dirac cones must come in pairs, is not applicable to the SDC state since the chiral symmetry is explicitly broken by the mass term.

Tight binding model: The silicene system is described by the four-band second-nearest-neighbor tight binding model ${ }^{16}$,

$$
\begin{aligned}
H & =-t \sum_{\langle i, j\rangle \alpha} c_{i \alpha}^{\dagger} c_{j \alpha}+i \frac{\lambda_{\mathrm{SO}}}{3 \sqrt{3}} \sum_{\langle\langle i, j\rangle\rangle \alpha \beta} \nu_{i j} c_{i \alpha}^{\dagger} \sigma_{\alpha \beta}^{z} c_{j \beta} \\
& +i \lambda_{\mathrm{R} 1}\left(E_{z}\right) \sum_{\langle i, j\rangle \alpha \beta} c_{i \alpha}^{\dagger}\left(\boldsymbol{\sigma} \times \hat{\boldsymbol{d}}_{i j}\right)_{\alpha \beta}^{z} c_{j \beta} \\
& -i \frac{2}{3} \lambda_{\mathrm{R} 2} \sum_{\langle i, j\rangle\rangle \alpha \beta} \mu_{i} c_{i \alpha}^{\dagger}\left(\boldsymbol{\sigma} \times \hat{\boldsymbol{d}}_{i j}\right)_{\alpha \beta}^{z} c_{j \beta} \\
& -\ell \sum_{i \alpha} \mu_{i} E_{z} c_{i \alpha}^{\dagger} c_{i \alpha},
\end{aligned}
$$

where $c_{i \alpha}^{\dagger}$ creates an electron with spin polarization $\alpha$ at site $i$, and $\langle i, j\rangle /\langle i, j\rangle\rangle$ run over all the nearest/next-nearest neighbor hopping sites. We explain each term. (i) The first term represents the usual nearest-neighbor hopping with the transfer energy $t=1.6 \mathrm{eV}$. (ii) The second term ${ }^{20}$ represents the effective SO coupling with $\lambda_{\text {SO }}=3.9 \mathrm{meV}$, where $\boldsymbol{\sigma}=\left(\sigma_{x}, \sigma_{y}, \sigma_{z}\right)$ is the Pauli matrix of spin, with $\nu_{i j}=+1$ if the next-nearest-neighboring hopping is anticlockwise and $\nu_{i j}=-1$ if it is clockwise with respect to the positive $z$ axis. 
(iii) The third term represents the first Rashba SO coupling associated with the nearest neighbor hopping, which is induced by external electric field. It satisfies $\lambda_{\mathrm{R} 1}(0)=0$ and becomes of the order of $10 \mu \mathrm{eV}$ at the critical electric field $E_{\mathrm{c}}=\lambda_{\mathrm{SO}} / \ell=17 \mathrm{meVA}^{-116}$. (iv) The forth term ${ }^{21}$ represents the second Rashba SO coupling with $\lambda_{\mathrm{R} 2}=0.7 \mathrm{meV}$ associated with the next-nearest neighbor hopping term, where $\mu_{i}= \pm 1$ for the A (B) site, and $\hat{\boldsymbol{d}}_{i j}=\boldsymbol{d}_{i j} /\left|\boldsymbol{d}_{i j}\right|$ with the vector $\boldsymbol{d}_{i j}$ connecting two sites $i$ and $j$ in the same sublattice. (v) The fifth term ${ }^{16}$ is the staggered sublattice potential term. Due to the buckled structure the two sublattice planes are separated by a distance, which we denote by $2 \ell$ with $\ell=0.23 \AA$. It generates a staggered sublattice potential $\propto 2 \ell E_{z}$ between silicon atoms at $\mathrm{A}$ sites and $\mathrm{B}$ sites in electric field $E_{z}$.

Low-energy Dirac theory: We analyze the physics of electrons near the Fermi energy, which is described by Dirac electrons near the $\mathrm{K}$ and $\mathrm{K}^{\prime}$ points. We also call them the $\mathrm{K}_{\eta}$ points with $\eta= \pm$. The effective Dirac Hamiltonian in the momentum space reads 16

$$
\begin{aligned}
H_{\eta}= & \hbar v_{\mathrm{F}}\left(\eta k_{x} \tau_{x}+k_{y} \tau_{y}\right)+\lambda_{\mathrm{SO}} \sigma_{z} \eta \tau_{z}-\ell E_{z} \tau_{z} \\
& +a \eta \tau_{z} \lambda_{\mathrm{R} 2}\left(k_{y} \sigma_{x}-k_{x} \sigma_{y}\right) \\
& +\lambda_{\mathrm{R} 1}\left(E_{z}\right)\left(\eta \tau_{x} \sigma_{y}-\tau_{y} \sigma_{x}\right) / 2
\end{aligned}
$$

where $\sigma_{a}$ and $\tau_{a}$ are the Pauli matrices of the spin and the sublattice pseudospin, respectively. The first term arises from the nearest-neighbor hopping, where $v_{\mathrm{F}}=\frac{\sqrt{3}}{2} a t=5.5 \times 10^{5} \mathrm{~m} / \mathrm{s}$ is the Fermi velocity with the lattice constant $a=3.86 \AA$. There is no recognizable effect from the term $\lambda_{\mathrm{R} 1}\left(E_{z}\right)$ as far as we have numerically checked. Although we include all terms in numerical calculations, in order to simplify the formulas and to make the physical picture clear, we set $\lambda_{\mathrm{R} 1}\left(E_{z}\right)=0$ in all analytic formulas.

There are four bands in the energy spectrum of $H_{\eta}$. The band gap is located at the $\mathrm{K}$ and $\mathrm{K}$ ' points, and given by $2\left|\Delta\left(E_{z}\right)\right|$, where ${ }^{16}$

$$
\Delta\left(E_{z}\right)=\eta s_{z} \lambda_{\mathrm{SO}}-\ell E_{z}
$$

with the spin $s_{z}= \pm 1$. It is a good quantum number at the K and K' points. The spin $s_{z}$ is an almost good quantum number even away from the $\mathrm{K}$ and $\mathrm{K}^{\prime}$ points because $\lambda_{\mathrm{R} 2}$ is a small quantity.

As $\left|E_{z}\right|$ increases, the gap decreases linearly, and closes at the critical point $\left|E_{z}\right|=E_{\mathrm{cr}}$ with

$$
E_{\mathrm{cr}}=\lambda_{\mathrm{SO}} / \ell=17 \mathrm{meV} / \AA
$$

and then increases linearly. Silicene is a semimetal due to gapless modes at $\left|E_{z}\right|=E_{\mathrm{cr}}$, while it is an insulator for $\left|E_{z}\right| \neq E_{\mathrm{cr}}$. It is to be noted that the change of the gap is suppressed by the screening effect due to the polarization of the A and B sublattices ${ }^{15}$. Even if the effect is taken into account, however, the gap changes linearly as a function of the external field. Hence, the present results remain true provided the external field is renormalized appropriately.

Photo-induced topological insulator: We consider a beam of circularly polarized light irradiated onto the silicene sheet.
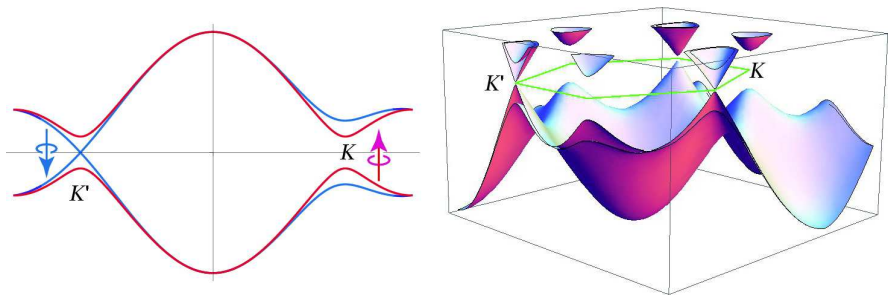

FIG. 1: (Color online) The band structure of a silicene in the SDC state. The gap is open at the $\mathrm{K}$ point with a parabolic dispersion but closed at the $\mathrm{K}$ ' point with a linear dispersion.

The corresponding electromagnetic potential is given by

$$
\boldsymbol{A}(t)=(A \sin \Omega t, A \cos \Omega t),
$$

where $\Omega$ is the frequency of light with $\Omega>0$ for the right circulation and $\Omega<0$ for the left circulation. The light intensity is characterized by the dimensionless number $\mathcal{A}=e A a / \hbar$, where $\mathcal{A}$ is typically less than 1 for intensity of lasers and pulses available in the frequency regime of our interests. The gauge potential satisfies the time-periodicity, $\boldsymbol{A}(t+T)=$ $\boldsymbol{A}(t)$, with $T=2 \pi /|\Omega|$. The electromagnetic potential is introduced into the Hamiltonian (2) by way of the minimal substitution, that is, replacing the momentum $\hbar k_{i}$ with the covariant momentum $P_{i} \equiv \hbar k_{i}+e A_{i}$.

A question arises whether a topological classification is possible in non-equilibrium situation, that is, when the Hamiltonian has an explicit time dependence. The answer is yes, provided the potential is time-periodic. A convenient method is to use the Floquet theory ${ }^{5-9}$. The topological classification is possible with the aid of the static effective Hamiltonian appropriately constructed.

We summarize the result of the floquet theory. When the light frequency is off-resonant for any electron transitions, light does not directly excite electrons and instead effectively modifies the electron band structures through virtual photon absorption processes. Such an off-resonant condition is satisfied for the frequency $\hbar|\Omega| \gg t$ in our model with $\pi$ bands. The influence of such off-resonant light is summarized $^{7}$ in the static effective Hamiltonian defined by $\Delta H_{\text {eff }}=(i \hbar / T) \log U$, where $U=\mathcal{T} \exp \left[-i / \hbar \int_{0}^{T} H(t) d t\right]$ is the time evolution operator with $\mathcal{T}$ the time-ordering operator. In the limit of $\mathcal{A} \ll 1, \Delta H_{\text {eff }}$ is particularly simple near the Dirac points: $\Delta H_{\mathrm{eff}}=(\hbar \Omega)^{-1}\left[H_{-1}, H_{+1}\right]+O\left(\mathcal{A}^{4}\right)$, where $H_{ \pm 1}$ is the Fourier component of Hamiltonian, that is, $H_{ \pm 1}=\frac{1}{T} \int_{0}^{T} H(T) e^{ \pm i t|\Omega|} d t$. The modification of the Hamiltonian due to the time-periodic perturbation is understood as the sum of two second-order processes, where electrons absorb and then emit a photon, and vice versa.

By explicitly calculating the commutation, we have the effective Hamiltonian, $H_{\text {eff }}^{\eta}=H_{\eta}+\Delta H_{\text {eff }}$, with

$$
\begin{array}{r}
\Delta H_{\mathrm{eff}}=-\frac{\mathcal{A}^{2}}{\hbar \Omega}\left[\left(\hbar v_{\mathrm{F}}\right)^{2} \eta \tau_{z}-\left(a \lambda_{\mathrm{R} 2}\right)^{2} \sigma_{z}\right. \\
\left.-a \lambda_{\mathrm{R} 2} \hbar v_{\mathrm{F}}\left(\eta \tau_{x} \sigma_{y}-\tau_{y} \sigma_{x}\right)\right] .
\end{array}
$$

It modifies the band structure. The modification is remarkable, which we demonstrate based on analytic formulas by 


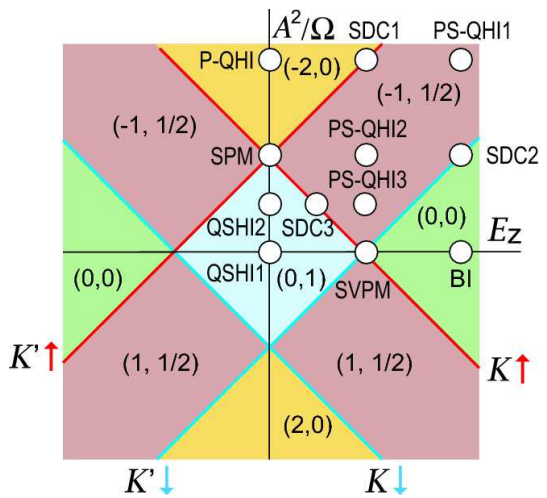

FIG. 2: (Color online) Phase diagram in the $\left(E_{z}, \mathcal{A}^{2} / \Omega\right)$ plane. A circle shows a point where the energy spectrum is calculated and shown in Fig 3 Heavy lines represent phase boundaries indexed by $\mathrm{K}_{\eta}$ and $s_{z}=\uparrow \downarrow$. There appear a SDC state along the line, which is characterized by a single gapless Dirac cone at the $\mathrm{K}_{\eta}$ point with spin $s_{z}$. The topological charges $\left(C, C_{s}\right)$ are also indicated.

neglecting the second Rashba terms $\left(\propto \lambda_{\mathrm{R} 2}\right)$ since $\lambda_{\mathrm{R} 2}$ is a small constant. The gap is given by $2\left|m_{\mathrm{D}}\right|$ with the Dirac mass,

$$
m_{\mathrm{D}}=\eta s_{z} \lambda_{\mathrm{SO}}-\ell E_{z}-\eta \hbar v_{\mathrm{F}}^{2} \mathcal{A}^{2} \Omega^{-1} .
$$

Hence we can control the Dirac mass by applying electric field $E_{z}$ and/or coherent laser beam $\propto \mathcal{A}^{2} / \Omega$. It is to be emphasized that the band gaps at the $\mathrm{K}$ and $\mathrm{K}^{\prime}$ points can be made different in general. We can make one Dirac cone gapless and the other Dirac cone gapped. This is the SDC state. The realization of different band gaps at the two valleys is an entirely new phenomenon. In Fig 1] we have illustrated the band structure of the SDC state calculated based on the tight-binding Hamiltonian (1) together with the inclusion of the Haldane term (10) we discuss later.

Spin-Chern number: Each topological phase is indexed by the topological quantum numbers, which are the Chern number $\mathcal{C}$ and the $\mathbb{Z}_{2}$ index. If the spin $s_{z}$ is a good quantum number, the $\mathbb{Z}_{2}$ index is identical to the spin-Chern number $\mathcal{C}_{s}$ modulo 2. They are defined by $\mathcal{C}=\mathcal{C}_{+}+\mathcal{C}_{-}$and $\mathcal{C}_{s}=\frac{1}{2}\left(\mathcal{C}_{+}-\mathcal{C}_{-}\right)$, where $\mathcal{C}_{ \pm}$is the summation of the Berry curvature in the momentum space over all occupied states of electrons with $s_{z}= \pm 1$. They are well defined even if the spin is not a good quantum number ${ }^{22.23}$. In the present model the spin is not a good quantum number because of spin mixing due to the Rashba couplings $\lambda_{R 1}$ and $\lambda_{R 2}$. A convenient way is to calculate them in the system without the Rashba couplings and then adiabatically switching on these couplings to recover the present system 22,23 .

When we set $\lambda_{\mathrm{R} 1}=\lambda_{\mathrm{R} 2}=0$, the Hamiltonian (2) becomes block diagonal. For each spin $s_{z}= \pm 1$ and valley $\eta= \pm$, it describes a two-band system in the form, $H=\boldsymbol{\tau} \cdot \boldsymbol{d}$, where $d_{x}=\eta \hbar v_{\mathrm{F}} k_{x}, d_{y}=\hbar v_{\mathrm{F}} k_{y}, d_{z}=m_{\mathrm{D}}$. The summation of the Berry curvature is reduced to the Pontryagin index in the two-band system ${ }^{2}$. They are determined uniquely by the Dirac mass and the valley index. We explicitly have $\mathrm{e}^{17}$

$$
\mathcal{C}_{s_{z}}^{\eta}=\frac{\eta}{2} \operatorname{sgn}\left(m_{\mathrm{D}}\right)
$$

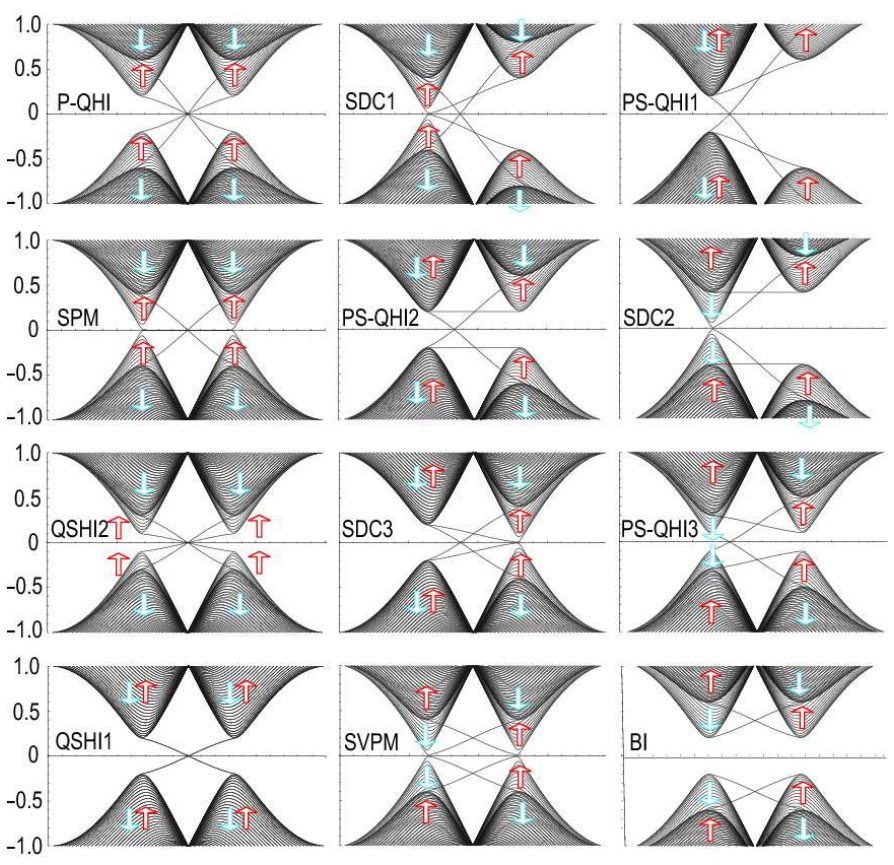

FIG. 3: (Color online) The photon-dressed band structure of a silicene nanoribbon at marked points in the phase diagram (Fig 2). The vertical axis is the energy in uit of $t$, and the horizontal axis is the momentum. We can clearly see the Dirac cones representing the energy spectrum of the bulk. Lines connecting the two Dirac cones are edge modes. The spin $s_{z}$ is practically a good quantum number, which we have assigned to the Dirac cones.

for the $\mathrm{K}_{\eta}$ valley. The Chern and spin-Chern numbers are given by $\mathcal{C}=\sum_{\eta= \pm}\left(\mathcal{C}_{+}^{\eta}+\mathcal{C}_{-}^{\eta}\right)$ and $\mathcal{C}_{s}=\sum_{\eta= \pm} \frac{1}{2}\left(\mathcal{C}_{+}^{\eta}-\mathcal{C}_{-}^{\eta}\right)$, which are shown in the phase diagram (Fig 2). The phase boundaries are given by $m_{\mathrm{D}}=0$ with (77). A topological phase transition occurs when the sign of the mass term changes. The topological numbers are $\left(C, C_{s}\right)=(0,0)$ in the $\mathrm{BI},(0,1)$ in the QSHI, $(-2,0)$ in the P-QHI, and $\left(-1, \frac{1}{2}\right)$ in the PS-QHI for $E>0$ and $\Omega>0$ in Fig 2 , In all these states the band gap is open, where the Fermi level is present, and they are insulators. We have derived these results without the Rashba interactions. They remain true when they are switched on adiabatically.

The Hall conductivity is given for each spin component by using the TKNN formula ${ }^{24}, \sigma_{x y}^{s_{z}}=e^{2} /(2 \pi \hbar) \sum_{\eta= \pm} \mathcal{C}_{s_{z}}^{\eta}$. The charge-Hall and spin-Hall conductivities are

$$
\sigma_{x y}=\sigma_{x y}^{\uparrow}+\sigma_{x y}^{\downarrow}, \quad \sigma_{x y}^{s}=\sigma_{x y}^{\uparrow}-\sigma_{x y}^{\downarrow} .
$$

They are equal to the Chern and spin-Chern numbers up to the normalization $e^{2} / 2 \pi \hbar$.

Photo-induced edge modes: A further insight follows from the fact that the commutator $\left[H_{-1}, H_{+1}\right]$ is interpreted as the second-neighbor hopping ${ }^{7}$. Hence, $\Delta H_{\text {eff }}$ is equivalent to the Haldane model ${ }^{25}$,

$$
\Delta H_{\mathrm{eff}}=-i \eta \hbar v_{\mathrm{F}}^{2} \mathcal{A}^{2} /(3 \sqrt{3} \Omega) \sum_{\langle\langle i, j\rangle\rangle \alpha \beta} \nu_{i j} c_{i \alpha}^{\dagger} c_{j \beta} .
$$

Based on this observation we have calculated the band structure of a silicene nanoribbon with zigzag edges, which we give 


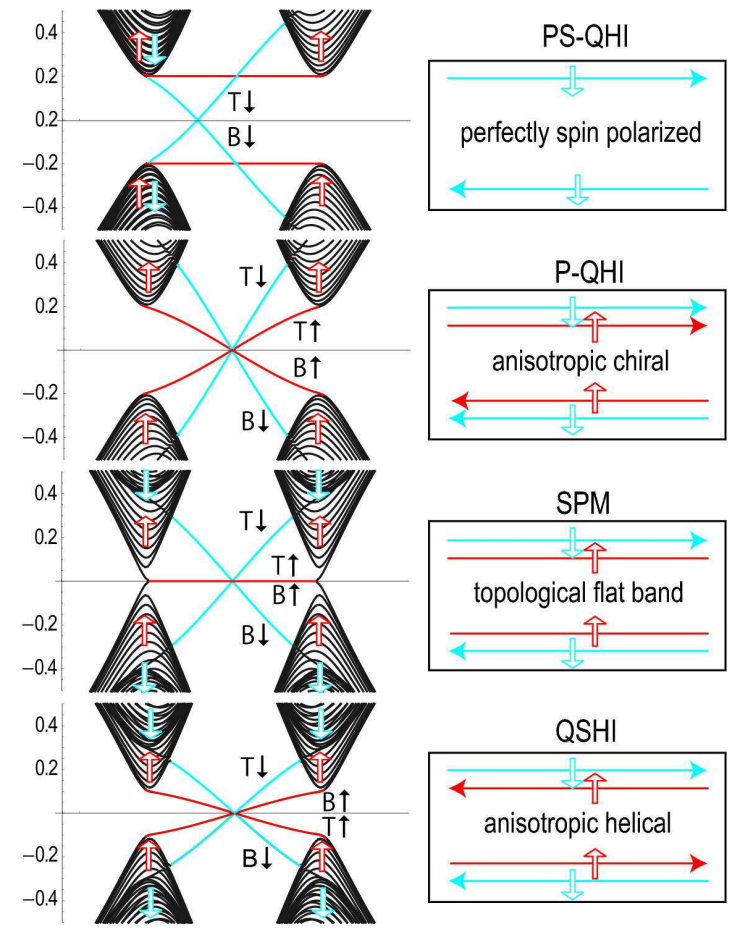

FIG. 4: (Color online) Enlarged edge states of silicene nanoribbons for the QSHI, SPM, P-QHI and PS-QHI. An anisotropic helical (chiral) current flows in QSHI (P-QHI), where the velocities of up and down spins are different in each edge. A topological flat band appears in SPM. Solid lines marked "T" represent edge modes propagating on the top edge, while dotted lines marked "B" represent edge modes propagating on the bottom edge.

in Fig 3 for typical points in the phase diagram (Fig 2). The edge mode changes between the chiral and helical states by the topological phase transition.

Conclusions: We have discovered a class of new phases by applying circular polorized light to silicene in the presence of electric field $E_{z}$, as summarized in the phase diagram (Fig 2) and in the band structures of associated nanoribbons (Fig 3).
We summarize their typical features.

The P-QHI exhibits quantum Hall effects without magnetics field. They have anisotropic chiral edge modes. It is remarkable that the velocities of up and down spins in chiral edge states are different, as found by the different slopes of the edge modes in Fig 4. This is not the case in graphene ${ }^{5}$. Similarly, the velocities of up and down spins in helical edge states are different in QSHI. The difference increases as the intensity of light increases.

The SPM appears at the critical point between the QSHI and the P-QHI. It is interesting to compare the state with the SVPM state appearing at the critical point between the QSHI and the BI. Due to the identical spin configuration at the $\mathrm{K}$ and $\mathrm{K}$ ' points, there emerge spin polarized topological flat bands in the SPM state (Fig, 4).

A particularly intriguing state is the SDC state emerging along the phase boundary, where, e.g., the gap is open at the $\mathrm{K}$ point but closed at the $\mathrm{K}$ ' point. The spin is up polarized at $\mathrm{K}$ point and down polarized at $\mathrm{K}^{\prime}$ point. Thus the net spin is polarized in the SDC state. Hence, this state is ferromagnet without magnetic field or exchange interactions. To create this state we have broken the time-reversal and space-inversion symmetries. We comment that there is no fermion doubling problem in the SDC state, because the chiral symmetry is explicitly broken by the mass term.

In this paper we have studied the second order effect in the photo coupling $\mathcal{A}$. When the off-resonant condition $\hbar|\Omega| \gg t$ is satisfied, there is no optical absorption. The lowest frequency is determined by the band width $3 t=4.8 \mathrm{eV}=$ $10^{15} \mathrm{~Hz}$. Below this frequency, the optical absorption occurs, which is the first order effect in $\mathcal{A}$. The peculiar optical selection rules and circular dichroism have already been predicted in this regime ${ }^{18}$.

I am very much grateful to N. Nagaosa and T. Oka for many helpful discussions on the subject. This work was supported in part by Grants-in-Aid for Scientific Research from the Ministry of Education, Science, Sports and Culture No. 22740196.
1 M.Z Hasan and C. Kane, Rev. Mod. Phys. 82, 3045 (2010).

2 X.-L. Qi and S.-C. Zhang, Rev. Mod. Phys. 83, 1057 (2011).

3 A. P. Schnyder, S. Ryu, A. Furusaki and A. W. W. Ludwig, Phys. Rev. B 78, 195125 (2008).

${ }^{4}$ W. Yao, A. H. MacDonald and Q. Niu, Phys. Rev. Lett 99, 047401 (2007).

5 T. Oka and H. Aoki, Phys. Rev. B 79, 081406(R) (2009).

6 J. Inoue and A. Tanaka, Phys. Rev. Lett. 105, 017401 (2010).

7 T. Kitagawa, T. Oka, A. Brataas, L. Fu, and E. Demler, Phys. Rev. B 84, 235108 (2011).

${ }^{8}$ N. Lindner, G. Refael and V. Gaslitski, Nat. Phys. 7, 490 (2011).

9 B. Dóra, J. Cayssol, F. Simon and R. Moessner, Phys. Rev. Lett. 108, 056602 (2012).

${ }^{10}$ P. Vogt, , P. De Padova, C. Quaresima, J. A., E. Frantzeskakis, M. C. Asensio, A. Resta, B. Ealet and G. L. Lay, Phys. Rev. Lett. 108, 155501 (2012).
11 C.-L. Lin, R. Arafune, K. Kawahara, N. Tsukahara, E. Minamitani, Y. Kim, N. Takagi, M. Kawai, Appl. Phys. Express 5, Art No. 045802 (2012) .

12 A. Fleurence, R. Friedlein, T. Ozaki, H. Kawai, Y. Wang, and Y. Yamada-Takamura, Phys. Rev. Lett. 108, 245501 (2012).

13 C.-C. Liu, W. Feng, and Y. Yao, Phys. Rev. Lett. 107, 076802 (2011).

14 M. Ezawa, New J. Phys. 14, 033003 (2012).

15 N. D. Drummond, V. Zólyomi, and V. I. Fal'ko, Phys. Rev. B 85, 075423 (2012).

${ }^{16}$ M. Ezawa, Phys. Rev. Lett 109, 055502 (2012).

17 M. Ezawa, Eur. Phys. J. B 85, 363 (2012).

18 M. Ezawa, Phys. Rev. B 86, 161407(R) (2012).

19 H. B. Nielsen and M. Ninomiya, Nucl. Phys. B 185, 20 (1981).

${ }^{20}$ C. L. Kane and E. J. Mele, Phys. Rev. Lett. 95, 226801 (2005); ibid 95, 146802 (2005). 
${ }^{21}$ C.-C. Liu, H. Jiang, and Y. Yao, Phys. Rev. B, 84, 195430 (2011).

22 E. Prodan, Phys. Rev. B 80, 125327 (2009).

${ }^{23}$ D. N. Sheng, Z. Y. Weng, L. Sheng and F. D. M. Haldane, Phys. Rev. Lett. 97036808 (2006).
${ }^{24}$ D. J. Thouless, M. Kohmoto, M. P. Nightingale, and M. den Nijs, Phys. Rev. Lett. 49, 405 (1982).

25 F. D. M. Haldane, Phys. Rev. Lett. 61, 2015 (1988). 